S. Kwok, M. Dopita, and R. Sutherland, eds.

\title{
Past and Future Evolution of Sakurai's Object
}

\author{
Falk Herwig \\ University of Victoria, Victoria, BC, Canada; \\ fherwig@uvastro.phys.uvic.ca
}

\begin{abstract}
We present a stellar evolution model sequence of the past and future evolution of the post-AGB born again star Sakurai's object (V $4334 \mathrm{Sgr}$ ). In order to match the short evolutionary time scale we have to assume that the convective ingestion of hydrogen-rich envelope material into the He-flash convection zone proceeds slower than predicted by the mixing length theory. For the future we predict a swift second evolution through the central star region before a second born-again evolution occurs.
\end{abstract}

The fast evolving born again asymptotic giant branch (AGB) star V 4334 Sgr provides a unique opportunity to test stellar evolution and nucleosynthesis models of the very late thermal pulse (VLTP) which occurs in about $10 \%$ of all central stars of planetary nebulae (CSPN) (Iben \& McDonald, 1995). V 4334 Sgr displayed an extraordinary fast evolution from the pre-white dwarf (WD) stage to the AGB in only three years (Duerbeck et al., 2000). In addition a peculiar realtime abundance evolution has been documented for this star by Asplund et al. (1999). We address both of these properties by exploring theoretical models. For example, Herwig \& Langer (2001) proposed a new nucleosynthesis mechanism for the lithium overabundance of V $4334 \mathrm{Sgr}$, because the well known hot-bottom burning mechanism which operates in massive AGB stars had to be excluded here. However, the latest VLTP stellar evolution model by Herwig et al. (1999) could not match the observed fast evolution of V 4334 Sgr although considerable effort had been put into a consistent numerical method for simultaneous mixing and nucleosynthesis. Herwig (2001) assumed that convective mixing of envelope material (cold fuel) into the He-flash convection zone is retarded with respect to the mixing-length theory (MLT) velocity. In that case the p-capture nuclear energy is released closer to the surface which leads to a faster inflation of the outermost layers. With this model the observed born again evolution time scale of V 4334 Sgr can be matched (Fig. 1). It should be noted, that MLT was never designed to apply to the ingestion of cold fuel into a convectively unstable nuclear burning zone.

The new model reproduces the $\mathrm{C}$-isotopic and the $\mathrm{C} / \mathrm{N}$ elemental ratio of Sakurai's object. Much more detailed information is available from the spectroscopic study of Asplund et al. (1999), and the observed iron-deficiency has already been addressed by Herwig, Lugaro \& Werner (these proceedings). An important feature of the new VLTP model sequence is the superposition of two born again phases (Fig. 1). The first, fast return to the AGB is driven by the $\mathrm{H}$-flash fueled by protons ingested into deeper layers from the envelope. After 


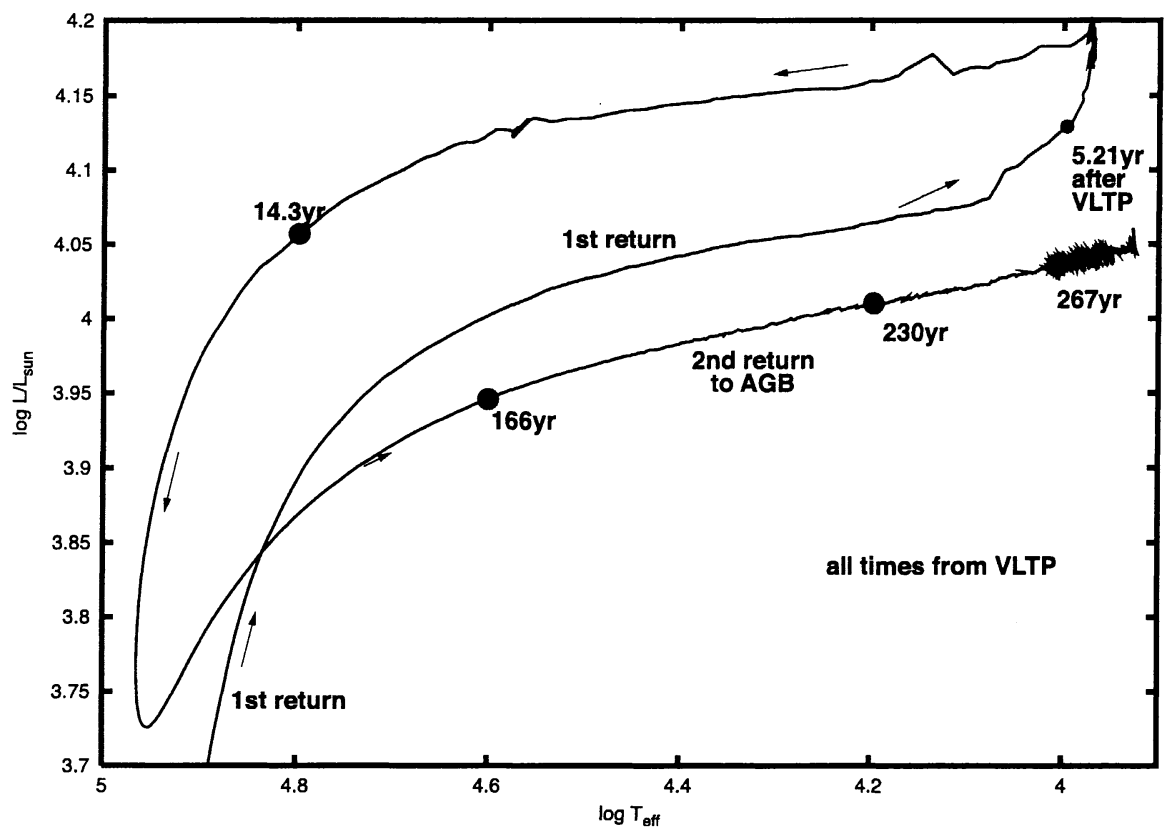

Figure 1. Post-AGB stellar evolution model sequence following a very late thermal pulse for comparison with Sakurai's object.

the star returns to the CSPN stage it is predicted to follow a second, He-flash driven born again evolution which proceeds on the more familiar time scale of the order of $\sim 100 \mathrm{yr}$. The current location of Sakurai's object is at the end of the $1^{\text {st }}$ return to the AGB. The predicted second evolution through the CSPN phase should be noticeable on a timescale of decades and we therefore expect that there is more to learn from Sakurai's object in our lifetime.

Acknowledgments. I would like to thank D.A. VandenBerg for support through his Operating Grant from the Natural Science and Engineering Research Council of Canada.

\section{References}

Asplund, M., Lambert, D. L., Kipper, T., Pollacco, D., \& Shetrone, M. D. 1999, A\&A, 343,507

Duerbeck, H. W., Liller, W., Sterken, C., et al. 2000, AJ, 119, 2360

Herwig, F. 2001, ApJ Lett., 554, L71

Herwig, F., Blöcker, T., Langer, N., \& Driebe, T. 1999, A\&A, 349, L5

Herwig, F. \& Langer, N. 2001, Nucl. Phys. A, 688, 221, astro-ph/0010120

Iben, Jr., I. \& MacDonald, J. 1995, in White Dwarfs, ed. D. Koester \& K. Werner, LNP No. 443 (Heidelberg: Springer), 48 